\title{
Molecular genetics of atypical teratoid/rhabdoid tumors
}

\author{
Jaclyn A. Biegel, Ph.D. \\ Division of Human Genetics, Department of Pediatrics, and Department of Pathology and Laboratory \\ Medicine, The Children's Hospital of Philadelphia and the University of Pennsylvania School of \\ Medicine, Philadelphia, Pennsylvania
}

\begin{abstract}
Rhabdoid tumors are extremely aggressive malignancies that generally occur in infants and young children. The most common locations are the kidney and central nervous system (atypical teratoid/rhabdoid tumor [RT]), although RTs can also arise in most soft-tissue sites. Rhabdoid tumors in all anatomical locations have a similar molecular origin. Mutation or deletion of both copies of the hSNF5/INI1 gene that maps to chromosome band 22q11.2 is observed in approximately $70 \%$ of primary tumors. An additional 20 to $25 \%$ of tumors have reduced expression at the RNA or protein level, indicative of a loss-of-function event. The INI1 protein is a component of the SWI/SNF chromatinremodeling complex. The complex is recruited to promoters of a large variety of genes involved in cell signaling, growth, and differentiation. This review summarizes what is currently known regarding the molecular genetics of RTs.
\end{abstract}

\section{KEY WORDS • rhabdoid tumor • hSNF5/INI1 • atypical teratoid/rhabdoid tumor • chromosome 22}

Rhabdoid tumors are highly malignant neoplasms that predominantly occur in infants and young children. The peak incidence is between birth and 3 years of age. Originally described as an aggressive tumor in the kidney, ${ }^{3}$ it is now apparent that an RT can arise in most soft-tissue sites, as well as the brain and spinal cord. ${ }^{26,32}$ The cell of origin is not known. Because they can exhibit divergent differentiation along neural, epithelial, and mesenchymal lines and can arise in multiple parts of the body, it is assumed that the cell of origin must be a primitive stem cell, possibly derived from the neural crest. $^{26}$

The most common locations for RTs are the kidney and the CNS. ${ }^{26,32}$ Central nervous system AT/RTs account for approximately 1 to $2 \%$ of pediatric brain tumors and approximately $10 \%$ of CNS tumors in infants. The majority of such tumors are identified in children between the 1st and 4 th years of life. They occur in both supratentorial and infratentorial locations, notably in the cerebellopontine angle..$^{32}$ Most AT/RTs are composed of rhabdoid cells juxtaposed to areas of primitive neuroepithelial tissue, which resembles a PNET and has mesenchymal and/or epithelial elements. This complex histological pattern has routinely led to misclassification of AT/RT - most often as PNET, but also as choroid plexus carcinoma, germ cell tumor, ependymoma, and glioblastoma. ${ }^{9,32}$ Positive staining of the rhabdoid cells with antibodies to epithelial membrane antigen can often be

Abbreviations used in this paper: AT/RT = atypical teratoid/rhabdoid tumor; $\mathrm{CNS}=$ central nervous system; $\mathrm{CPC}=$ choroid plexus carcinoma; FISH = fluorescence in situ hybridization; INI1 = integrase interactor- 1 : LOH $=$ loss of heterozygosity; PNET $=$ primitive neuroectodermal tumor; RT = rhabdoid tumor; RT-PCR = reverse transcription-polymerase chain reaction. used to distinguish AT/RTs from other CNS malignancies. ${ }^{32}$ Although AT/RTs are often refractory to therapy, some success in treating them has been achieved with surgery, radiation, and myeloablative chemotherapy with stem cell rescue. ${ }^{13}$ The tumors often initially respond to chemotherapy, but patients quickly relapse, exhibiting signs of both local and metastatic disease. Current estimates suggest a 2-year survival rate of $15 \%$ for children suffering from AT/RT. Compared with the fatal outcome for most patients harboring AT/RTs, the 5-year survival rate for a standard-risk medulloblastoma-PNET is much higher, at approximately $85 \%$. An accurate diagnosis is therefore essential so that appropriate therapy can be given.

Because RTs are generally considered tumors of infancy, their occurrence in adults has been called into question. The presence of rhabdoid cells alone is not a sufficient criterion on which to make the diagnosis, as these cells may be present in a variety of solid tumors. Composite RTs of the CNS or soft tissues demonstrate rhabdoid cells but otherwise have little in common with the typical childhood RT. ${ }^{27} \mathrm{Re}-$ cently, we have confirmed the occurrence of RTs in the CNS in several adult patients by providing cytogenetic and molecular evidence for inactivation of INII..$^{29}$ The availability of molecular and immunohistochemical assays to distinguish the clinically aggressive RT from other benign or malignant tumors should allow clinical oncologists to tailor treatment appropriately.

The recognition of AT/RT as a distinct pathological entity has been supported by findings of cytogenetic studies, which demonstrated that RTs had minimal alterations and did not contain the isochromosome 17q typical of medulloblastoma/PNETs. ${ }^{6}$ Rhabdoid tumors of the brain are most 
often characterized by loss of one copy of the entire chromosome 22 (monosomy 22) or a deletion or translocation specifically involving chromosome band 22q11.2. Rhabdoid tumors of the kidney also demonstrate deletions of chromosome 22. As for AT/RTs, the deletions can be submicroscopic and are best identified by FISH studies. ${ }^{7,8}$ Softtissue extrarenal RTs are most often characterized by translocations of chromosome 22, although in almost every case, the translocation results in a submicroscopic deletion at the translocation breakpoint. ${ }^{7}$ Interstitial deletion in the cytogenetically normal-appearing chromosome 22 leads to homozygous deletion of the RT locus. Loss of one copy of chromosome 22 , with concomitant duplication of the remaining allele or mitotic recombination, results in $\mathrm{LOH}$ for alleles on chromosome 22 and is typically observed in tumors with cytogenetically normal karyotypes for which FISH studies demonstrated normal findings. The identification of deletions involving chromosome band 22q11.2 implied the presence of a tumor suppressor gene for RTs. Positional cloning strategies ultimately resulted in the identification of $h S N F 5 / I N I 11 / B A F 47 / S M A R C B 1^{20,24}$ as a rhabdoid tumor suppressor gene for renal and soft-tissue RTs. ${ }^{39}$ We first described the occurrence of germline and somatic mutations of INII in CNS AT/RTs, confirming the hypothesis that RTs in different sites had a common molecular origin. ${ }^{8}$

The INI1 protein is one of at least 10 in the BAF (BRGassociated factor) or SWI/SNF complex, ${ }^{28,42}$ an adenosine triphosphate-dependent chromatin-remodeling complex. ${ }^{34}$ Each complex contains INI1 and either the adenosine triphosphatase member Brg1 or Brm, so INI1 is therefore expressed in all normal cells at all stages of development. Structural studies have demonstrated that the SWI/SNF complex disrupts the nucleosome by removing DNA from the histone octomer surface. ${ }^{2}$ The complex can interact with multiple DNA sites, a function consistent with the possibility that nucleosomes at distal regulatory elements, such as enhancers and promoters, could simultaneously be disrupted. The remodeling of chromatin by SWI/SNF complexes facilitates transcriptional activation and repression, although the mechanisms related to the targeting of SWI/SNF complexes to specific regions of the genome (for example, promoters) are still not understood. At present, it is unclear whether INI1 might have distinct functions independent of its role as a member of the SWI/SNF complex.

Several in vitro studies have suggested that INI1 suppresses tumor formation by regulation of the p16-CDK4/ cyclin D-Rb-E2F pathway (reviewed in Imbalzano and Jones $^{14}$ ). Introduction of INII into INII-deficient RT cell lines results in flat cell morphology, changes in the actin cytoskeleton, accumulation of cells in $\mathrm{G}_{0} / \mathrm{G}_{1}$, and, as found in

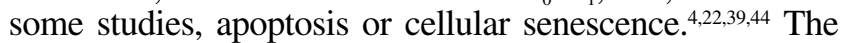
mechanism by which INI1 activates p16 is not completely understood. It appears to act in a cell type-specific manner to recruit the SWI/SNF complex to the p16 promoter. Increased expression of p16 leads to inhibition of the cyclin D1-CDK4 complex, retaining Rb in its hypophosphorylated antiproliferative state. ${ }^{25}$ Whereas expression of p16 appears to be necessary for INI1-associated suppression of cell growth, expression of cyclin D1 appears to be necessary for tumor development. ${ }^{38}$ Both of these genes are markers of cell proliferation, and thus may not be the direct targets of INI1 responsible for tumor initiation. Vries, et al. ${ }^{41}$ have recently suggested that inactivation of INI1 may lead to polyploidy and chromosomal instability, due to abnormal chromosomal segregation. ${ }^{41} \mathrm{I}$, my colleagues, and others have shown that primary RTs are usually diploid and have minimal regions of $\mathrm{LOH}^{6,8}$ It is possible that the chromosomal instability noted in the studies reported by Vries, et al., ${ }^{41}$ is related to the nature of the experimental system that was used.

Murine knockout models have provided functional evidence for Inil/Snf5 as a tumor-suppressor gene. ${ }^{12,21,30}$ The Inil/Snf5 null mutants die in the periimplantation stage in embryogenesis. Directly or through its function in the SWI/ SNF complex, INi1 represses or activates a large number of genes required for early stage development. Gresh, et al.,"11 recently inactivated Inil in the developing liver, which led to early postnatal lethality. Mice that are Inil/Snf5 haploinsufficient develop tumors that resemble RTs histologically, but these tumors arise most often in soft tissues, often on the face. In contrast to those in humans, murine tumors rarely develop in the brain or kidney. Roberts and coworkers $^{31}$ have constructed a conditional knockout of Inil/Snf5 in which mice developed rapidly lethal tumors that resembled mature T-cell lymphomas. Interestingly, coinactivation of p16Ink $4 a$ or $R b$ did not have a significant effect on tumor formation, presumably because this pathway has already been activated by Inil/Snf5 loss. Coinactivation of $p 53$ led to a dramatic decrease in the time to tumor development, ${ }^{15}$ however, indicating that alterations in other pathways, such as DNA damage and repair, may impact tumorigenesis and perhaps response to therapeutic agents. Zhang, et al. ${ }^{44} \mathrm{dem}-$ onstrated that the introduction of INII into an INII-deficient cell line repressed transcription of cyclin D1. Using an immunohistochemical approach, they showed that RTs with inactivation of INII expressed the cyclin D1 protein. In recent studies, Tsikitis, et al. ${ }^{38}$ developed Ini1 ${ }^{+/-}$heterozygous mice that spontaneously develop RTs, yet crossing these mice with cyclin D1-deficient mice abrogated tumor predisposition. By 15 months, $25 \%$ of the Ini1 ${ }^{+1-}$ Cyclin $\mathrm{DI}^{+/+}$or Cyclin $\mathrm{DI}^{+/-}$mice developed tumors, whereas none of the Inil ${ }^{+/-}$Cyclin $\mathrm{Dl}^{-/-}$mice developed tumors. Tsikitis, et al., propose that cyclin D1 expression is necessary for RT growth and thus may be a good target for therapy. Because the cyclin D1-Rb pathway is one of the key modulators of cell cycle progression, the absence of cyclin D1 in the progenitor cells may not allow these cells to become fully transformed and give rise to detectable tumors. That does not negate the possibility, however, that one or more upstream genes may be the initial targets for INI1induced tumorigenesis.

Consistent with the proposed characterization of INII as a tumor suppressor gene, a deletion and mutation analysis of CNS have demonstrated biallelic alterations of INII in both renal and extrarenal RTs. ${ }^{7,840}$ Tumors have been shown to harbor a homozygous deletion of INII or a deletion of one copy of the INII gene with a mutation in the remaining homologue. The majority of the mutations are point or frameshift changes that introduce a novel stop codon, and thus predict premature truncation of the protein.

A summary of the deletion and mutation studies for 119 RTs studied in the author's laboratory follows and is highlighted in Figs. 1 and 2. The data represent the results from 79 brain and spinal cord tumors, 25 renal tumors, and nine extrarenal RTs, as well as brain and renal or brain and soft- 


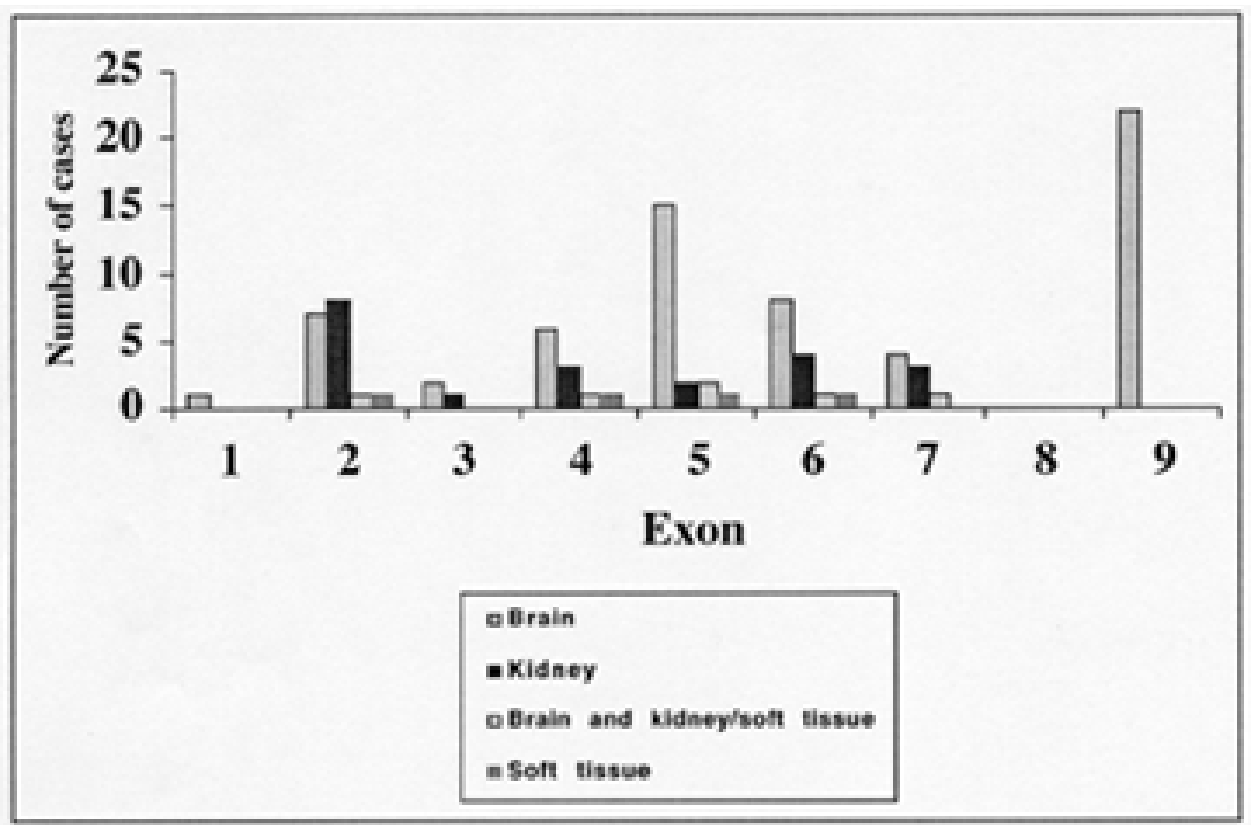

Fig. 1. Bar graph depicting the distribution of 96 coding-sequence mutations in the INII gene.

tissue RTs in six children. Twenty-six tumors had homozygous deletions of INII, including $16(20 \%)$ of the brain and spinal cord tumors, four (17\%) of the kidney tumors, and six $(67 \%)$ of the extrarenal RTs. Coding-sequence mutations were detected in $63(80 \%)$ of the brain tumors, 21 $(84 \%)$ of the kidney tumors, and three $(33 \%)$ of the softtissue tumors, as well as in brain and renal or extrarenal RTs in six children (100\%). The distribution of coding-sequence mutations among the nine exons of the INII gene is shown in Fig. 1. Two tumors of the brain and one soft-tissue tumor had two different mutations, presumably on different alleles. The tumors with single mutations had a loss of the wild-type allele, due either to a deletion, mitotic recombination, or loss and duplication of the mutant allele.

As shown in Figs. 1 and 2, we have not yet detected any mutations in exon 8, and exons 1 and 3 are greatly underrepresented. Exons 5 and 9 appear to be hotspots for the CNS tumors. Coding-sequence mutations in exon 2 were common among the renal RTs. As shown in Fig. 2, 48 of the 96 mutations were single-base pair-point mutations. All but one of these were nonsense mutations, predicting premature truncation of the protein. One missense mutation was detected in exon 9 (G1130A; arginine $\rightarrow$ histidine). In exon 5, a common C601T mutation was found in a total of 12 tumors, including 10 brain tumors, one renal tumor, and one patient with primary tumors of the brain and lung. It is likely that a germline mutation occurred in the latter, but we did not have normal tissue for confirmation. A C472T mutation was found in a total of seven tumors, including three brain tumors, three renal tumors (two in the germline), and a tumor of the skin. The C472T mutation does not appear to be associated with a particular site, but it is often observed in the germline.

There were four intragenic deletions of one or two exons, seven duplications (four to 19 bases), six insertions (one to 28 bases), and 31 deletions. The most frequent deletion was in exon 9, which accounted for a total of 20 of the mutations, and all in tumors of the brain or spinal cord. Of note, we have not yet detected this mutation in the blood of any of the patients. Fourteen of the mutations involved a deletion of one of four cytosines in bases 1145 to 1148 . The remaining six mutations were single deletions of one of two guanines at position 1143 or 1144 . Both mutations are predicted to cause a frame shift, with addition of an extra 96 amino acids to the end of the protein. If expressed, however, the protein is unstable, as it was not detected by Western blot analysis of three tumors or by immunohistochemical analysis with an INI1 antibody.

The majority of RTs in all sites demonstrate deletions and/or mutations of INII. In a substantial number of cases, there is no coding-sequence mutation, but quantitative RTPCR analysis demonstrates low expression of INI1. Hypermethylation of the INII promoter could result in reduced expression of INI1 in the absence of a coding-sequence mutation; however, identification of the promoter region and methylation studies demonstrated that the INII promoter region is unmethylated..$^{43}$ Genomic rearrangements, such as inversions, intragenic deletions, or translocations of 22q11.2 could result in loss of expression of INI1 and would be difficult to detect using PCR-based approaches. Alternative epigenetic modifications, disruption of binding to an enhancer sequence, or a second RT locus that may function upstream of INII could also lead to reduced INI1 expression.

Although it is clear that patients with INII alterations and/or a diagnosis of RT have an extremely poor prognosis, it is not apparent that the type of genetic alteration affects overall survival. At present, we have the clinical follow-up information for 45 children with AT/RTs. Although there was no apparent association of mutation with outcome, these patients underwent a variety of treatments, making it difficult to discern the contribution of genotype to outcome. Oncologists attempt to avoid radiation therapy to the brain in children younger than 3 years of age, which 


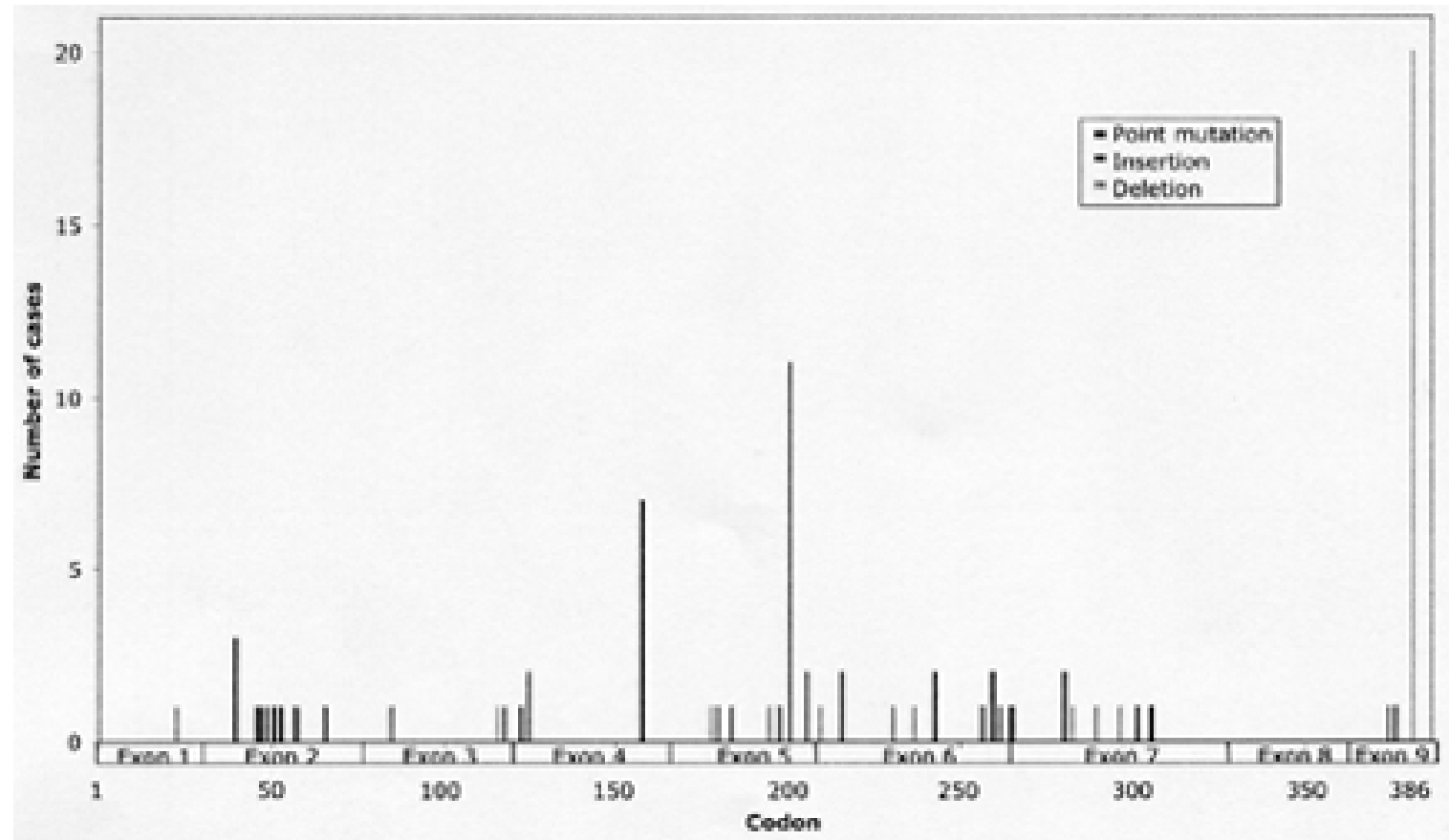

Fig. 2. Graph depicting distribution of INII mutations in RTs.

could affect the overall survival of this study population. An association of mutation with prognosis can best be elucidated in the context of a therapeutic trial that will control for treatment effects. Such a trial is currently in development with the Children's Oncology Group.

To develop accurate risk estimates for tumor development, one of our continuing goals is to determine the frequency of germline mutations in patients with RTs of the CNS, kidney, and/or soft tissues. The expectation is that children who present with more than one primary tumor, or who have a family history of RT, are most likely to have a germline INI1 mutation. Patients with soft-tissue tumors would be the least likely to demonstrate a germline mutation, because the tumors are most often homozygously deleted for INII.

To date, we have obtained matched blood and/or samples of second primary tumor tissue from 49 patients with RTs and coding-sequence mutations in INII. As shown in Table 1 , we identified germline mutations in $16(33 \%)$ of the 49 individuals, two of whom were siblings. This estimate of the risk of germline mutation may be high because physicians and parents may have been more likely to contribute blood samples once a mutation was identified in the tumor tissue. This aspect may be balanced, however, by the fact that alterations resulting in decreased expression of INI1 at the RNA and protein level that have not yet been elucidated may also be present in the germline. Twenty percent of AT/RTs and $67 \%$ of extrarenal RTs have homozygous deletions of INII as identified by FISH studies. The extrarenal soft-tissue tumors generally showed homozygous deletions due to deletion at the breakpoint of an apparently cytogenetically balanced chromosome 22 translocation and a submicroscopic deletion in the remaining chromosome 22 homologue. We hypothesize that some of these patients may have constitutional INII deletions below the level detected by the FISH assays currently in use.

There were several notable findings among the patients with germline mutations. First, as expected, children with two primary tumors were most likely to have germline mutations (five of six patients). The frequency of these mutations was higher in the children with renal tumors (six of $11 ; 55 \%$ ) compared with children with brain or spinal cord tumors (five of 27; 18\%). The lower frequency of germline mutations in children with brain tumors may be biased by the high frequency of exon 9 mutations that appear to be of somatic origin and the high rate of homozygous deletion.

As shown in Table 1, germline coding-sequence mutations have been detected in exons 2, 4, 5, 6, and 7. All of the mutations result in the introduction of a novel stop codon. Little is known regarding the structural function of the protein, and, interestingly, all of the mutations appear to result in loss of protein expression. Two single base-pair mutations were seen in two individuals each. The C472T in exon 4 and C601T in exon 5 both result in arginine $\rightarrow$ stop mutations, and, in fact, cytosine $\rightarrow \mathrm{T}$ transitions predominated. Most interesting, however, is the fact that we have not yet detected the most frequently occurring mutations, the single $\mathrm{G}$ or $\mathrm{C}$ deletion in exon 9, in any patient with an RT of the CNS. This finding suggests that a dominant negative effect of this mutation may exist that is incompatible with a term pregnancy.

Patients with germline mutations of INII are predisposed to RTs of the brain, kidney, and soft tissues and may present with more than one primary tumor. These tumors are most often identified within the 1st year of life, and tend to have the worst prognosis. ${ }^{33}$ Reports of familially associated cases of AT/RT in the literature are, however, limited. Sevenet, et al., ${ }^{36}$ reported on three families with multiple affected 
Atypical teratoid/rhabdoid tumors

TABLE 1

Germline mutations of the INII gene in patients with RTs

\begin{tabular}{lccll}
\hline \hline \multicolumn{1}{c}{ Location } & Exon & Codon & \multicolumn{1}{c}{ Mutation } & Parent of Origin \\
\hline brain & 2 & 51 & G152A & unknown \\
brain & 4 & 123 & C367T & mother \\
brain & 4 & 125 & 373 dup 4 bp & mother \\
brain & 5 & 201 & C601T & mother \\
kidney & 2 & 47 or 48 & $141 / 4$ ins C & mother \\
kidney & 2 & 153 & C157T & unknown \\
kidney & 4 & 158 & C472T & unknown \\
kidney & 4 & 158 & C472T & unknown \\
kidney & 5 & 206 & G617A & unknown \\
kidney & 7 & 266 & 797 del 10bp & unknown \\
brain \& lung & 5 & 201 & C601T & unknown \\
brain \& kidney & 2 & 66 & C197A & mother \\
brain \& kidney & 5 & 198 & C592T & unknown \\
brain \& kidney & 6 & 257 & C769T & mother \\
brain \& kidney & 7 & 297 & 889 del 7bp & unknown \\
\hline
\end{tabular}

siblings who were shown to carry germline mutations of INII. None of the parents carried the mutation; thus, gonadal mosaicism was implicated as a potential mechanism to explain the familial predisposition to these tumors. IzyckaSwieszewska, et al., ${ }^{16}$ reported the occurrence of an AT/RT in an infant, with simultaneous presentation of a PNET of the spinal cord in the child's father. Although the father's tumor revealed a homozygous missense mutation at codon 174 in the TP53 gene that was not present in the child, a full analysis of the INII gene was not performed in either the child or her father.

To date, authors of only two reports discuss an inherited INII mutation. ${ }^{17,37}$ In the family reported by Taylor, et al., ${ }^{37}$ a child with AT/RT inherited a germline exon 7 splice site mutation in the INII gene from her unaffected mother, a carrier. The mother's brother carried the identical mutation and had died as a child after diagnosis of a CPC. We have recently studied a family with two half brothers who presented at ages 2 and 17 months with cerebral AT/RT. ${ }^{17}$ The maternal uncle was reported to have had a medulloblastoma and a renal RT, and he died at 2 years of age. The two children carried the identical germline mutation in exon 4 of the INII gene, which was also inherited from the unaffected mother. The affected uncle was a half sibling, and thus the grandmother is also likely to be the carrier of the same INII mutation. The occurrence of a second family with unaffected carriers supports the hypothesis that the risks of an RT developing in the context of a germline mutation may be variable. It is possible that the risk of RT decreases with age, in contrast to germline mutations of TP53 or BRCA. This age-related risk may be better understood once the cell of origin for RTs is identified. It is also interesting to note that the mutation was inherited from the mother in both of these families. Although the frequency of deletions or $\mathrm{LOH}$ of the paternal or maternal allele appear to be equal in patients with sporadic RTs, we have noted a bias in the parent of origin of the mutated allele in children with germline INI1 mutations. In our series, six of six germline mutations were in the maternal allele.

The ability to study the effect of coding-sequence mutations or altered RNA expression on protein expression has been greatly facilitated by our development of an immunohistochemical assay for INI1. ${ }^{19}$ We have shown that all RTs with coding-sequence mutations or reduced RNA expression demonstrate loss of nuclear expression in the tumor cells. Furthermore, a majority of histologically confirmed RTs without mutations of INII also showed loss of protein expression. Most important, we have shown the clinical utility of this assay for distinguishing AT/RTs from tumors with overlapping histological features, such as PNETs, choroid plexus carcinomas, and rhabdoid meningiomas. ${ }^{18,19,27}$

Normal cells exhibit nuclear expression of INI1; thus, infiltrating lymphocytes, blood vessels, and fibroblasts serve as an internal positive control. Our first set of experiments was designed to determine if loss of INI1 expression, as detected by immunohistochemical analysis, was correlated with INII deletion and mutation. ${ }^{19}$ We analyzed 20 AT/RTs that had either a homozygous deletion or mutation of INII or a decreased expression of INI1 as determined by quantitative RT-PCR. All 20 tumors showed retained expression of INI1 in blood vessels and infiltrating lymphocytes, with loss of expression in the tumor cells. The antibody recognizes an epitope between amino acids 257 and 359 in the C terminus of the protein. We expected that tumors with $5^{\prime}$ mutations would show loss of protein expression and were surprised to find that all mutations led to loss of protein expression, including those in tumors with an exon 9 delC or delG mutation that was predicted to result in the addition of 96 amino acids to the end of the protein. These results were confirmed by Western blot analysis, which also demonstrated loss of the INI1 protein in tumors with exon $9 \mathrm{mu}-$ tations. Ten tumors with classic features of medulloblastoma/PNET all showed retained expression of INI1. This finding was consistent with results of our previous mutation studies that did not demonstrate INII mutations in these tumors. ${ }^{5}$ The most interesting cases in this study involved a series of seven tumors with histological features or immunophenotypic profiles that were equivocal for PNET (particularly the anaplastic or large-cell variant) or that carried an initial diagnosis of AT/RT but had no mutation of INII. All seven tumors retained nuclear staining of INI1, consistent with a revised diagnosis of medulloblastoma/PNET. In at least two cases, the identification of cytogenetic abnormalities (such as isochromosome 17q and MYC amplification) typically seen in medulloblastoma/PNET was used to support the diagnosis.

The remaining 23 tumors in this study ${ }^{18}$ included a variety of histological subtypes. Only one tumor, an oligodendroglioma, demonstrated loss of INI1 expression, but there was insufficient material for mutation analysis, suggesting that loss of INI1 is specific to AT/RTs. This assay also appears to be more sensitive than mutation detection for distinguishing AT/RTs from other malignancies. To date, we have analyzed 26 tumors with classic histological features of AT/RTs that have loss of protein expression and no INII mutation. Loss of INI1 expression in the absence of a mutation may identify a subgroup of tumors with epigenetic modification of the INI1 locus or alterations in another gene that could modify INI1 expression.

The most controversial tumor type with respect to INII mutation status has been CPC. Sevenet, et al., ${ }^{35}$ initially reported that four of six CPCs had mutations of INII. Gessi, et al. ${ }^{10}$ have also suggested that 40 to $50 \%$ of CPCs have mutations in INII. Distinguishing CPCs from AT/RTs on histological grounds alone can be difficult in cases that show overlapping features (that is, extensive epithelial dif- 
ferentiation within an AT/RT or limited epithelial differentiation in a CPC) because the immunoprofiles of these two tumors overlap extensively. We obtained a series of 28 tumors with an initial diagnosis of CPC from seven different institutions and the Cooperative Human Tissue Network. ${ }^{18}$ Twenty-one of the 28 cases had classic features of CPC, and all retained expression of INI1. Seven of the tumors demonstrated loss of expression of INI1. An independent review of the 28 tumors was performed by several expert neuropathologists who had no knowledge of the results of the immunohistochemical analysis. The seven tumors that tested negative for INI1 expression were either reclassified as AT/RTs, or had features of the tumors that suggested AT/RT. We therefore concluded that a loss of INII is specific for AT/ RTs, and that CPCs with INII mutations have been classified as such due to the difficult nature of distinguishing these two entities. ${ }^{18}$

Combined mutation analysis and INI1 immunohistochemical assays have also been useful in elucidating the nature of AT/RTs seen in the setting of progressive disease. We recently studied an optic pathway ganglioglioma that evolved into an AT/RT in an 11-year-old child after 10 years of treatment. ${ }^{1}$ A mutation in exon 9 was identified in formalin-fixed tissue from the composite AT/RT-ganglioglioma. Expression of INI1 detected by immunohistochemical analysis was found in the ganglioglioma component and normal cells only and was absent in the RT cells. The transformation to AT/ RT was probably due to acquisition of the INII mutation, resulting in a therapy-resistant and ultimately fatal tumor.

Similar to CNS malignancies, renal and extrarenal RTs are characterized by loss of protein expression of INI1 as determined by immunohistochemical assays, the use of which has shown the greatest clinical utility in distinguishing soft-tissue RTs from other malignancies. Epithelioid sarcomas are a complex histological group of tumors that occur in both children and adults. A recognized subgroup, termed "proximal epithelioid sarcoma," has a multinodular pattern of growth, rhabdoid cells, and an aggressive course - features that overlap with RTs. Cytogenetic and $\mathrm{LOH}$ studies have demonstrated loss of sequences in 22q. We previously characterized two epithelioid sarcomas with homozygous deletions of INII in an adult and in a child (unpublished data). In both cases, the pathologists revised the diagnosis to RT. Modena, et al., ${ }^{23}$ recently identified homozygous deletions of INII in five of 11 epithelioid sarcomas, all of the proximal type. They suggest that INII alterations may not be specific for RTs but highlight the fact that no mutations of INII were identified. This scenario raises at least two possibilities: 1) that the proximal type of epithelioid sarcoma is a variant of RT, with a similar genetic origin and clinical course, and 2) that a locus close to INII is involved in the development of proximal epithelioid sarcomas (and that INII is deleted by virtue of its proximity to that locus). Both possibilities can be addressed by analyzing a larger series of tumors.

Deletion and mutation analyses of INI1, as well as functional studies of the gene, are of interest for several reasons. From the clinical diagnostic standpoint, we need to supplement routine histological evaluation with molecular genetic analysis to identify patients who need extremely aggressive therapy to enhance survival. The identification of germline INII mutations in patients with AT/RTs of the CNS is a basis for beginning to understand the molecular origin of what had been considered a sporadic pediatric tumor. We will be able to determine if family members or cancer survivors are at risk for developing RTs. From a basic science perspective, understanding the role of INII as a member of the SWI/SNF complex in tumorigenesis will be critical for identifying downstream target genes and, ultimately, for designing biologically based therapeutic strategies.

\section{References}

1. Allen JC, Judkins AR, Rosenblum MK, et al: Atypical teratoid/ rhabdoid tumor evolving from an optic pathway ganglioglioma: case study. Neurooncol 8:79-82, 2006

2. Bazett-Jones DP, Cote J, Landel CC, et al: The SWI/SNF complex creates loop domains in DNA and polynucleosome arrays and can disrupt DNA-histone contacts within these domains. Mol Cell Biol 19:1470-1478, 1999

3. Beckwith JB, Palmer NF: Histopathology and prognosis of Wilms tumors: results from the First National Wilms' Tumor Study. Cancer 41:1937-1948, 1978

4. Betz BL, Strobeck MW, Reisman DN, et al: Re-expression of hSNF5/INI1/BAF47 in pediatric tumor cells leads to G1 arrest associated with induction of p16ink4a and activation of RB. Oncogene 21:5193-5203, 2002

5. Biegel JA, Fogelgren B, Zhou JY, et al: Mutations of the INI1 rhabdoid tumor suppressor gene in medulloblastomas and primitive neuroectodermal tumors of the central nervous system. Clin Cancer Res 6:2759-2763, 2000

6. Biegel JA, Rorke LB, Packer RJ, et al: Monosomy 22 in rhabdoid or atypical teratoid tumors of the brain. J Neurosurg 73: 710-714, 1990

7. Biegel JA, Tan L, Zhang F, et al: Alterations of the hSNF5/INII gene in central nervous system atypical teratoid/rhabdoid tumors and renal and extrarenal rhabdoid tumors. Clin Cancer Res 8: 3461-3467, 2002

8. Biegel JA, Zhou JY, Rorke LB, et al: Germ-line and acquired mutations of INI1 in atypical teratoid and rhabdoid tumors. Cancer Res 59: 74-79, 1999

9. Burger PC, Yu IT, Tihan T, et al: Atypical teratoid/rhabdoid tumor of the central nervous system: a highly malignant tumor of infancy and childhood frequently mistaken for medulloblastoma: a Pediatric Oncology Group study. Am J Surg Pathol 22:1083-1092, 1998

10. Gessi M, Giangaspero F, Pietsch T: Atypical teratoid/rhabdoid tumors and choroid plexus tumors: when genetics "surprise" pathology. Brain Pathol 13: 409-414, 2003

11. Gresh L, Bourachot B, Reimann A, et al: The SWI/SNF chromatin-remodeling complex subunit SNF5 is essential for hepatocyte differentiation. EMBO J 24:3313-3324, 2005

12. Guidi CJ, Sands AT, Zambrowicz BP, et al: Disruption of Ini1 leads to peri-implantation lethality and tumorigenesis in mice. Mol Cell Biol 21:3598-3603, 2001

13. Hilden JM, Meerbaum S, Burger P, et al: Central nervous system atypical teratoid/rhabdoid tumor: results of therapy in children enrolled in a registry. J Clin Oncol 22:2877-2884, 2004

14. Imbalzano AN, Jones SN: Snf5 tumor suppressor couples chromatin remodeling, checkpoint control and chromosomal stability. Cancer Cell 7:294-295, 2005

15. Isakoff MS, Sansam CG, Evans JA, et al: Inactivation of the Snf5 tumor suppressor stimulates cell cycle progression and cooperates with p53 loss in oncogenic transformation. Proc Natl Acad Sci U S A 102:17745-17750, 2005

16. Izycka-Swieszewska E, Debiec-Rychter M, Wasag B, et al: A unique occurrence of a cerebral atypical teratoid/rhabdoid tumor in an infant and a spinal canal primitive neuroectodermal tumor in her father. J Neurooncol 61:219-225, 2003

17. Janson K, Nedzi LA, David O, et al: Predisposition to atypical 
teratoid/rhabdoid tumor due to an inherited INI1 mutation, in Pediatr Blood Cancer, 2005, (http://dx.doi.org/10.1002/pbc. 20622)

18. Judkins AR, Burger PC, Hamilton RL, et al: INI1 protein expression distinguishes atypical teratoid/rhabdoid tumor from choroid plexus carcinoma. J Neuropath Exp Neurol 64: 391-397, 2005

19. Judkins AR, Mauger J, Rorke LB, et al: Immunohistochemical analysis of hSNF5/INI1 in pediatric CNS neoplasms. Am J Surg Pathol 28: 644-650, 2004

20. Kalpana GV, Marmon S, Wang W, et al: Binding and stimulation of HIV-1 integrase by a human homolog of yeast transcription factor SNF5. Science 266:2002-2006, 1994

21. Klochendler-Yeivin A, Fiette L, Barra J, et al: The murine SNF5/INI1 chromatin remodeling factor is essential for embryonic development and tumor suppression. EMBO Rep 1: 500-506, 2000

22. Medjkane S, Novikov E, Versteege I, et al: The tumor suppressor hSNF5/INI1 modulates cell growth and actin cytoskeleton organization. Cancer Res 64:3406-3413, 2004

23. Modena P, Lualdi E, Facchinetti F, et al: SMARCB1/INII tumor suppressor gene is frequently inactivated in epithelioid sarcomas. Cancer Res 65:4012-4019, 2005

24. Muchardt C, Sardet C, Bourachot B, et al: A human protein with homology to Saccharomyces cerevisiae SNF5 interacts with the potential helicase hbrm. Nucleic Acids Res 23: 1127-1132, 1995

25. Oruetxebarria I, Venturini F, Kekarainen T, et al: P16INK4a is required for hSNF5 chromatin remodeler-induced cellular senescence in malignant rhabdoid tumor cells. J Biol Chem 279: 3807-3816, 2004

26. Parham DM, Weeks DA, Beckwith JB: The clinicopathologic spectrum of putative extrarenal rhabdoid tumors. An analysis of 42 cases studied with immunohistochemistry or electron microscopy. Am J Surg Pathol 18:1010-1029, 1994

27. Perry A, Fuller CE, Judkins AR, et al: INI1 expression is retained in composite rhabdoid tumors, including rhabdoid meningiomas. Mod Pathol 18:951-958, 2005

28. Phelan ML, Sif S, Narilikar GJ, et al: Reconstitution of a core chromatin remodeling complex from SWI/SNF subunits. Mol Cell 3: 247-253, 1999

29. Raisanen J, Biegel JA, Hatanpaa KJ, et al: Chromosome 22q deletions in adult atypical teratoid/rhabdoid tumors. Brain Pathol 15:23-28, 2005

30. Roberts CW, Galusha SA, McMenamin ME, et al: Haploinsufficiency of Snf5 (integrase interactor 1) predisposes to malignant rhabdoid tumors in mice. Proc Natl Acad Sci U S A 97: 13796-13800, 2000

31. Roberts CW, Leroux MM, Fleming MD, et al: Highly penetrant, rapid tumorigenesis through conditional inversion of the tumor suppressor gene Snf5. Cancer Cell 2: 415-425, 2002

32. Rorke LB, Packer RJ, Biegel JA: Central nervous system atyp- ical teratoid/rhabdoid tumors of infancy and childhood: definition of an entity. J Neurosurg 85:56-65, 1996

33. Savla J, Chen TT, Schneider NR, et al: Mutations of the hSNF5/ INI1 gene in renal rhabdoid tumors with second primary brain tumors. J Natl Cancer Inst 92: 648-650, 2000

34. Schnitzler GR, Sif S, Kingston RE: A model for chromatin remodeling by the SWI/SNF family. Cold Spring Harb Symp Quant Biol 63:535-543,1998

35. Sevenet N, Lellouch-Tubiana A, Schofield D, et al: Spectrum of hSNF5/INII somatic mutations in human cancer and genotypephenotype correlations. Hum Mol Genet 8:2359-2368, 1999

36. Sevenet N, Sheridan E, Amram D, et al: Constitutional mutations of the hSNF5/INI1 gene predispose to a variety of cancers. Am J Hum Genet 65:1342-1348, 1999

37. Taylor MD, Gokgoz N, Andrulis IL, et al: Familial posterior fossa brain tumors of infancy secondary to germline mutation of the hSNF5 gene. Am J Hum Genet 66: 1403-1406, 2000

38. Tsikitis M, Zhang Z, Edelman W, et al: Genetic ablation of Cyclin D1 abrogates genesis of rhabdoid tumors resulting from Ini1 loss. Proc Natl Acad Sci U S A 102:12129-12134, 2005

39. Versteege I, Medjkane S, Rouillard D, et al: A key role of the hSNF5/INI1 tumour suppressor in the control of the G1-S transition of the cell cycle. Oncogene 21:6403-6412, 2002

40. Versteege I, Sevenet N, Lange J, et al: Truncating mutations of hSNF5/INI1 in aggressive paediatric cancer. Nature 394: 203-206, 1998

41. Vries RG, Bezrookove V, Zuijderduijn LM, et al: Cancer-associated mutations in chromatin remodeler hSNF5 promote chromosomal instability by compromising the mitotic checkpoint. Genes Dev 19:665-670, 2005

42. Wang W, Xue Y, Zhou S, et al: Diversity and specialization of mammalian SWI/SNF complexes. Genes Dev 10:2117-2130, 1996

43. Zhang F, Tan L, Wainwright LM, et al: No evidence for hypermethylation of the hSNF5/INI1 promoter in pediatric rhabdoid tumors. Genes Chromosomes Cancer 34: 398-405, 2002

44. Zhang ZK, Davies KP, Allen J, et al: Cell cycle arrest and repression of cyclin D1 transcription by INI1/hSNF5. Mol Cell Biol 22:5975-5988, 2002

Manuscript received October 26, 2005.

Accepted in final form December 2, 2005.

This work was supported by a grant from the National Institutes of Health (CA46274) and the Brain Tumor Resource Laboratory of the Children's Oncology Group.

Address reprint requests to: Jaclyn A. Biegel, Ph.D., Room 1002, Abramson Research Building, The Children's Hospital of Philadelphia, 3615 Civic Center Boulevard, Philadelphia, Pennsylvania 19104.email:biegel@mail.med.upenn.edu. 